\title{
Flavor decomposition with precision pion and kaon cross sections
}

\author{
Tanja Horn*t \\ Catholic University of America and Jefferson Lab \\ E-mail: horntecua.edu
}

\begin{abstract}
Key properties of the nucleon are encoded in the correlation between their longitudinal momentum and their transverse position, such as the orbital angular momentum. This correlation is expressed through Generalised Parton Distributions (GPDs) [1, 2, 3, 4], which can be understood as spatial densities at different values of the longitudinal momentum of the quark, and due to the space-momentum correlation information encoded in the GPDs can link through the Ji sum rule to a parton's angular momentum [5]. Transverse Momentum-dependent parton Distributions (TMDs) are functions of both the longitudinal and transverse momentum of partons, and they offer a momentum tomography of the nucleon complementary to the spatial tomography of GPDs [6]. The GPD and TMD programs are at the heart of the scientific motivation of the $12 \mathrm{GeV}$ Jefferson Lab [7]. Recent data and prospects for deep exclusive pion and kaon electroproduction are presented, which conceptually would allow for flavour separations. Experimental tests of our theoretical understanding of the reaction mechanism are shown including longitudinal-transverse separated charged-pion cross section data and ratios. The prospects to use projected charged- and neutral pion data to further determine the spin, charge-parity and flavour of GPDs, including the helicity-flip GPDs, are discussed.
\end{abstract}

XXV International Workshop on Deep-Inelastic Scattering and Related Subjects 3-7 April 2017

University of Birmingham, $U K$

\footnotetext{
*Speaker.

† Supported in part by NSF grants PHY1306227 and PHY1306418
} 


\section{Introduction}

Generalised Parton Distributions (GPDs) encode the correlation between the quark/gluon transverse position in the nucleon and its longitudinal momentum, and can be measured in exclusive scattering processes at large $Q^{2}$, in which the nucleon is observed intact in the final state. It is recognized that Deeply Virtual Compton Scattering (DVCS) and Deeply Virtual Meson Production (DVMP) are two powerful processes to probe GPDs. Together they offer a path to a full 3-dimensional tomography of the nucleon structure.

\section{Validation of the exclusive reaction mechanism}

The key to extracting GPDs from experiment are QCD factorisation theorems [8, 9], which allow the amplitudes for deep exclusive processes to be expressed in terms of GPDs [2, 5]. GPD measurements at Jefferson Lab rely on the assumption that deep exclusive reactions are well described by their leading twist mechanism. The value of $Q^{2}$ at which this approximation is valid experimentally needs to be determined and the contributions of higher twist components to observables need to be quantified. The $Q^{2}$ dependence of cross sections is the only unambiguous way to separate higher twist contributions to deep exclusive channels.

Deeply Virtual Compton Scattering is the cleanest or golden channel to study GPDs. As the DVCS process interferes with the Bethe-Heitler process, one can access the DVCS amplitudes. At leading twist and leading order, one determines Compton Form Factors, which are integrals of GPDs over Bjorken $x$ with a kernel to describe the hard photon-quark interaction. Present analyses assume dominance of several GPDs, validity of twist-2 dominance, and a leading-order formalism. To go beyond this, one has to fully disentangle Compton scattering, Bethe-Heitler contributions, and their interference (after subtracting the known Bethe-Heitler contribution).

DVCS cross sections and polarised asymmetries can provide detailed and precise information about GPDs, but are sensitive only to a particular flavour combination. Exclusive meson production provides key additional information allowing the separation of different quark and anti-quark flavours [3]. The theoretical description of these processes is more complicated, and thus measurements that provide information about the reaction mechanism, e.g., tests of hard-soft factorisation, are essential. In particular, the emerging transversity GPDs $[10,11,12]$ may be accessed if dominance of the transverse cross section at small values of $t<0.3 \mathrm{GeV}^{2}$ can be experimentally verified. First measurements were carried out at $6 \mathrm{GeV}$ JLab by the DVCS Hall A Collaboration and a substantial contribution of the transverse $\pi^{0}$ cross section was found [13, 14].

To validate the meson factorisation theorems and potentially extract flavour separated GPDs from experiment, one has measure the separated longitudinal (L) and transverse (T) cross sections and their $t$ and $Q^{2}$ dependencies. Only L/T separated cross sections can unambigously show the dominance of longitudinal or transverse photons and allow one to determine possible correlations in $t$ and $Q^{2}$. The onset of factorisation for light mesons may be expected earlier than for heavier ones. Recent calculations predict the onset for pions and kaons in the $5-10 \mathrm{GeV}^{2}$ regime, a region accessible with planned $12 \mathrm{GeV}$ Jefferson Lab (JLab) experiments [15, 16, 17]. Thus, if meson factorisation is to be observed it is most probable for pion and kaon and it can be realized in the next few years. 


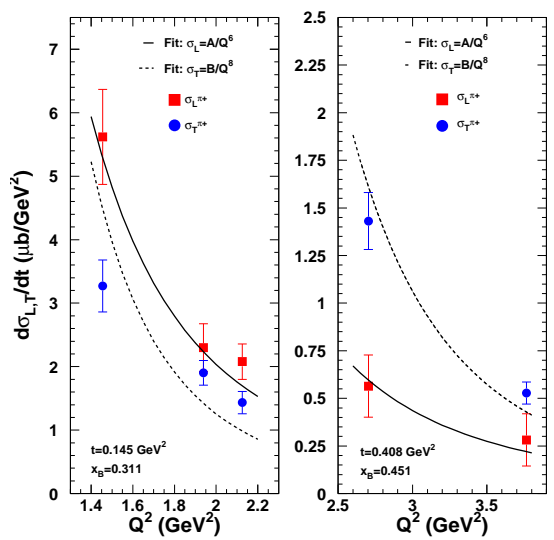

Figure 1: The $Q^{2}$-dependence of the separated cross sections at fixed values of $-t$ and $x_{B}$. The error bars denote the statistical and systematic uncertainties combined in quadrature. The solid and dashed curves show fits of the form $Q^{-6}$, for $\sigma_{L}$ and $Q^{-8}$ for $\sigma_{T}$, the predicted and expected $Q C D$ factorisation behaviour with $Q^{2}$.

\section{Experiments with SHMS in Hall $\mathrm{C}$ at Jefferson Lab $12 \mathrm{GeV}$}

Measuring L/T separated cross sections places strong demands on experimental facilities requiring rigorous control over systematic uncertainties. Hall $\mathrm{C}$ at JLab with its precision focusing spectrometers and particle identification detectors is the only facility available for carrying out these measurements. Two experiments are planned with the $12 \mathrm{GeV}$ JLab extending charged pion data to $Q^{2}$ of about $9 \mathrm{GeV}^{2}$ [16] and kaon data to $5.5 \mathrm{GeV}^{2}$ [17].

First measurements were carried out with at $6 \mathrm{GeV}$ JLab in Hall $\mathrm{C}$ and established the techniques and demonstrated the feasibility of measuring the $Q^{2}$ dependence of $\mathrm{L} / \mathrm{T}$ separated pion and kaon cross sections at fixed $x$ and $t$ as illustrated in Fig. 1. The $12 \mathrm{GeV}$ upgrade at Jefferson Lab (JLab 12) features new instrumentation that allows for pushing precision pion and kaon measurements to the highest momentum transfers to date. Planned experiments aim for precision measurements of charged pion data to $Q^{2}$ of about $9 \mathrm{GeV}^{2}$ [16] and kaon data to $5.5 \mathrm{GeV}^{2}$ [17]. These measurements are made possible by the combination of the two moderate acceptance, magnetic spectrometers in Hall $\mathrm{C}$ and particle identificatio detectors like aerogel Cherenkov detectors for kaon-proton separation [18]. The "High Momentum Spectrometer" (HMS) provides angular acceptance of $6 \mathrm{msr}$ and can detect particles with momenta up to $7 \mathrm{GeV} / \mathrm{c}$. The new "Super-High Momentum Spectrometer" (SHMS) features a solid angle of about $4 \mathrm{msr}$, a momentum coverage up to $11 \mathrm{GeV} / \mathrm{c}$, and covers scattering angles between 5.5 and 40 degrees. The small scattering angle capability, combined with excellent control of systematic uncertainties, kinematic reproducibility and well-understood acceptance, enables precision measurements of cross-sections and L/T separations at high luminosity $\left(>10^{38} / \mathrm{cm}^{2} \mathrm{~s}\right)$. Such data will provide access to the $\mathrm{L} / \mathrm{T}$ separated pion and kaon cross sections on a domain of momenta that is nearly four-times larger than that explored so far. The phase space covered by E12-07-105 is shown in Fig. 2.

E12-07-105 [16] aims primarily to probe conditions for factorisation of deep exclusive measurements for charged pions (the simplest reaction beyond DVCS). E12-07-105 will provide L/T 


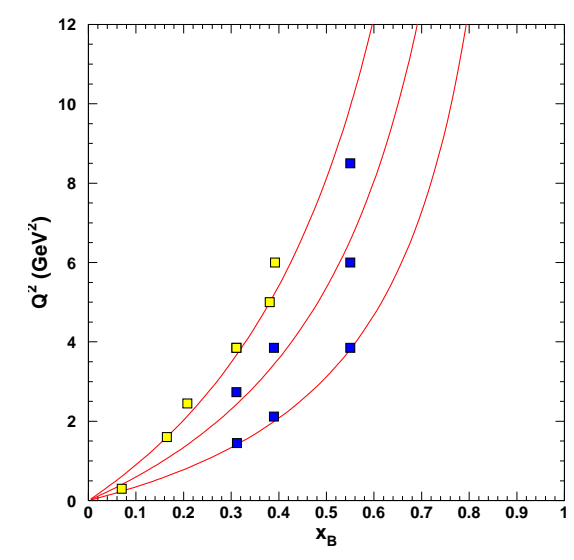

Figure 2: The kinematic phase space covered by $12 \mathrm{GeV}$ exclusive pion electroproduction experiments. The red lines indicate fixed $W$ values. The points denote precision measurements at fixed values of $Q^{2}$ and $x$

separated pion cross sections up to $Q^{2} \sim 9 \mathrm{GeV}^{2}$, which can be used to verify the applicability of the GPD formalism and to constrain theoretical models. This experiment will also extend pion form factor data up to the highest possible momentum transfers achievable at a $12 \mathrm{GeV}$ Jefferson Lab $Q^{2}=8.5 \mathrm{GeV}^{2}$. These data would allow for confirming recent calculations tied to dynamical chiral symmetry breaking, and thus could contribute a major step forward towards our understanding of QCD.

E12-09-011 [17] will provide the first L/T separated kaon cross sections beyond the resonance region to $Q^{2}=5.5 \mathrm{GeV}^{2}$. This will for the first time allow tests of the reaction mechanism in the transition from hadronic to partonic degrees of freedom when strangeness is in play. The data may also allow extractions of the kaon form factor at lower values of $t$ than previously accessible. The anticipated results will provide the most precise spatial mapping of the kaon to date and may reveal new insight on the role of the strange quark.

\section{New Opportunities for Hadron Structure Studies at Jefferson Lab 12 GeV}

The Neutral Particle Spectrometer (NPS) is a envisioned as a facility in Hall C, utilizing the well-understood HMS and the SHMS infrastructure, to allow for precision (coincidence) cross section measurements of neutral particles $\left(\gamma\right.$ and $\pi^{0}$ ). The NPS consists of a segmented crystalline scintillator-based calorimeter preceded by a sweeping magnet. This design provides detection down to minimum central angles of $7.2^{\circ}$ at a position of $4 \mathrm{~m}$ from the target, with $2-3 \mathrm{~mm}$ spatial and $1-2 \%$ energy resolution.

The NPS scientific program includes 4 fully approved experiments to date, which comprises about $20 \%$ of the beam time in Hall C. It enables precision measurements of the deeply-virtual Compton scattering cross section at different beam energies to extract the real part of the Compton form factor without any assumptions [19]. It makes possible measurements of the basic semiinclusive neutral-pion cross section to validate QCD factorisation, a cornerstone of 3D transverse momentum imaging [20]. It further allows measurements to push the energy scale of real Compton scattering, the process of choice to explore factorisation in a whole class of wide-angle processes, 
and its extension to neutral pion photo-production. Ideas exit for future experiments and new scientific directions taking advantage if the compatibility of NPS with Hall infrastructure. These include experiments with polarised targets and high-intensity photon sources.

The combination of high precision calorimetry with NPS and a novel compact high intensity photon source (CPS) [21] greatly enhances scientific benefit to exclusive processes like wide-angle and time-like Compton scattering with transverse polarized targets. It offers a gain in scientific production of a factor of 30 as compared to existing techniques. A similar compact high-intensity photon source technique would allow a gain of two decades to produce a $K_{L}$ beam photon source, which would open new avenues for hadron spectroscopy. The CPS includes a strong magnet after the radiator that deflects the exiting electrons, a long-bore collimator that lets the photon beam through. In this design, no photon tagging is needed as opposed to the Tagger Magnet concept and the magnet itself is the electron beam dump. Hermetic shielding around the setup and close to the source limits prompt radiation and activation. A $30 \mathrm{~kW}$ configuration was proven by simulation and heat dissipation calculations. Larger space available in Hall $\mathrm{D} / K_{L}$ allows for twice that beam power.

The combination of NPS and CPS allows for measurements of polarisation observables in Wide-Angle Compton Scattering, in particular, explicit, model-independent tests of factorisation by measuring the $s$-dependence of the polarisation obervables at fixed centre of mass angle. Measurements of the polarisation observable $A_{L L}$ at large angles allows for tests of the relevant degrees of freedom in hard exclusive reactions. Furthermore, the extraction of Axial and Pauli form factors from Wide-Angle Compton Scattering allows for constraining the GPDs $\widetilde{H}$ and $\mathrm{E}$ at high $t$.

\section{Summary}

Precision fully L/T separated cross section measurements are essential for the GPD programme at $12 \mathrm{GeV} \mathrm{JLab}$ allowing for validation of hard-soft factorisation in exclusive processes required for accessing the GPDs. The charged pion/kaon and neutral particle physics programme in Hall C plays an important role in the GPD programme. The Neutral Particle Spectrometer (NPS) gives the unique opportunity for coincidence precision cross section measurements with neutral particle final states. The combination of NPS and a novel Compact Photon Source and modern polarized targets opens new avenues for hadron structure studies.

\section{References}

[1] M. Diehl, Eur. Phys. J. C 25, 233 (2002); Phys. Rept. 388, 41 (2003).

[2] A.V. Radyushkin, Phys. Lett. B 385, 333 (1996); Phys. Lett. B 380, 417 (1996).

[3] K. Goeke, M.V. Polyakov and M. Vanderhaeghen, Prog. Part. Nucl. Phys. 47, 401 (2001)

[4] A.V. Belitsky and A.V. Radyushkin, Phys. Rept. 418, 1 (2005).

[5] X. Ji, Phys. Rev. D 55, 7114 (1997); Phys. Rev. Lett.78, 610 (1997).

[6] M Burkardt, Nucl. Phys. A 711 (2002) 27; Int. J. Mod. Physics A 18 (2003) 173.

[7] J. Dudek, R. Ent, R. Essig, K. Kumar, C. Meyer, R. McKeown, Z-E. Meziani, G.A. Miller, M. Pennington, D. Richards, L. Weinstein, G. Young, S. Brown, Eur. Phys. J. A48 (2012) 187. 
[8] J.C. Collins, L. Frankfurt, M. Strikman, Phys. Rev. D 56, 2982 (1997).

[9] J.C. Collins, A. Freund, Phys. Rev. D 59, 074009 (1999).

[10] S. V. Goloskokov and P. Kroll, Eur. Phys. J. C 65, 137 (2010) [arXiv:0906.0460 [hep-ph]].

[11] S. V. Goloskokov and P. Kroll, Eur. Phys. J. A 47, 112 (2011) [arXiv:1106.4897 [hep-ph]].

[12] G. R. Goldstein, J. O. Gonzales, Hernandez, S. Liuti, J. Phys. G 39 (2012) 115001.

[13] M. Defurne et al., Phys. Rev. Lett. 118 (2017) no. 22, 222002.

[14] M. Mazouz et al., Phys. Rev. Lett. 117 (2016) no. 26, 262001.

[15] T. Horn, C.D. Roberts, J. Phys. G43 (2017) no.7, 073001.

[16] T. Horn, G. Huber, et al., [Hall C Pion Factorization (P12) Collaboration],Approved JLab experiment E12-07-105: Scaling Study of the L-T Separated Pion Electroproduction Cross Section at $11 \mathrm{GeV}$. Available online: http://www.jlab.org/exp_prog/proposals/07/PR12-07-103.pdf

[17] T. Horn, G. Huber, P. Markowitz, et al., [Hall C Kaon Factorization (K12) Collaboration], Approved JLab experiment E12-09-011: Studies of the L-T Separated Kaon Electroproduction Cross Section from 5-11 GeV.

Available online: http://www.jlab.org/exp_prog/proposals/09/PR12-09-011.pdf

[18] T. Horn et al., Nucl. Instrum. Meth. A842 (2017) 28.

[19] C. Munoz-Camacho, T. Horn, C. Hyde, R. Paremuzyan, J. Roche, et al., and the Neutral Particle Spectrometer Collaboration, Approved Jefferson Lab experiment E12-13-010: Exclusive Deeply Virtual Compton and Neutral Pion Cross-Section Measurements in Hall C.

Available online: https://www.jlab.org/exp_prog/proposals/13/PR12-13-010.pdf

[20] R. Ent, T. Horn, E. Kinney, H. Mkrtchyan, V. Tadevosyan, et al., and the Neutral Particle Spectrometer Collaboration, Approved Jefferson Lab experiment E12-13-007: Measurement of Semi-Inclusive $\pi^{0}$ Production as Validation of Factorization.

Available online: https://www.jlab.org/exp_prog/proposals/13/PR12-13-007.pdf

[21] S. Ali et al. (NPS Collaboration), arxiv:1704.00816 (2017). 